\title{
Microwave-enhanced folding and denaturation of globular proteins
}

\author{
Bohr, Henrik; Bohr, Jakob
}

Published in:

Physical Review E. Statistical, Nonlinear, and Soft Matter Physics

Link to article, DOI:

10.1103/PhysRevE.61.4310

Publication date:

2000

Document Version

Publisher's PDF, also known as Version of record

Link back to DTU Orbit

Citation (APA):

Bohr, H., \& Bohr, J. (2000). Microwave-enhanced folding and denaturation of globular proteins. Physical Review E. Statistical, Nonlinear, and Soft Matter Physics, 61(4), 4310-4314. https://doi.org/10.1103/PhysRevE.61.4310

\section{General rights}

Copyright and moral rights for the publications made accessible in the public portal are retained by the authors and/or other copyright owners and it is a condition of accessing publications that users recognise and abide by the legal requirements associated with these rights.

- Users may download and print one copy of any publication from the public portal for the purpose of private study or research.

- You may not further distribute the material or use it for any profit-making activity or commercial gain

- You may freely distribute the URL identifying the publication in the public portal 


\title{
Microwave-enhanced folding and denaturation of globular proteins
}

\author{
Henrik Bohr and Jakob Bohr \\ Institute of Physics, Building 307, The Technical University of Denmark, DK-2800 Lyngby, Denmark
}

(Received 3 February 1999; revised manuscript received 20 July 1999)

\begin{abstract}
It is shown that microwave irradiation can affect the kinetics of the folding process of some globular proteins, especially $\beta$-lactoglobulin. At low temperature the folding from the cold denatured phase of the protein is enhanced, while at a higher temperature the denaturation of the protein from its folded state is enhanced. In the latter case, a negative temperature gradient is needed for the denaturation process, suggesting that the effects of the microwaves are nonthermal. This supports the notion that coherent topological excitations can exist in proteins. The application of microwaves hold promises for a wide range of biotechnological applications, such as protein synthesis, protein aggregation, etc., and may have implications for biological systems as well.
\end{abstract}

PACS number(s): 87.10.+e, 87.50.-a, 05.70.Ln

\section{INTRODUCTION}

The complex process of protein folding has many interesting facets $[1-4]$. One interesting aspect that has not been much addressed is to what extent external radiation can cause conformational effects. We suggest that microwaves may cause such effects through a stimulation of the coherent intrinsic dynamics in biomolecular chain molecules, e.g., DNA, RNA, and proteins. Such behavior is consistent with the view that protein folding is the result of a competition between torsional and bending forces, and not only driven by entropy $[5,6]$. Folded structures that are stabilized by hydrogen bonds, disulfide bridges, etc., can maintain a significant fraction of folding in balance with unfolded chains, and the biological evolution of living organisms selects proteins with the ability to acquire stable conformations.

We have previously suggested that, because of topological constraints, folded and unfolded proteins can possess an intrinsic dynamics with a collective nature [6]. The lower eigenmodes are in the microwave range of approximately a few $\mathrm{GHz}$, and the molecules may therefore be excitable by microwave irradiation. Because such intrinsic dynamics is important for the stability and folding of proteins, it is expected that one should be able to influence and change the protein folding and denaturation processes by applying microwave or ultrasound to protein solutions.

In this paper we present data from an experiment that gives support for such an effect, which we will discuss here on the basis of a theory about topological excitations of biomolecules. The experiment concerns refolding of a common protein in a denaturant solution to which microwave radiation is applied. The refolding of the protein, $\beta$-lactoglobulin, is monitored by the optical rotation dispersion (ORD) technique, which measures the polarization.

Previously, microwave effects have been reported for studies of bond breaking in plasmid DNA [7], in a method that has been developed for rapid and selective peptide-bond cleavage of proteins in a weak acidic solution [8,9], and in immunostaining of cytopathology specimens [10]. It has been claimed that these effects include some component that is nonthermal.

\section{EXPERIMENTAL PROCEDURE}

The pure $\beta$-lactoglobulin protein is dissolved in a urea solution with the stoichiometry of $5 \mathrm{mg}$ protein in $3 \mathrm{ml} 0.1 \mathrm{M}$ $\mathrm{KCI}$ and $4 \mathrm{ml} 4 M$ urea. The solution is then adjusted with $\mathrm{HCl}$ to have a $p \mathrm{H}$ around 2 .

The protein in this solution can be in three states: a cold denaturation state, a folded state, and a hot denaturation state, depending on temperature. Roughly $3 \mathrm{ml}$ of the sample is injected into the inner tube of the sample holder of a polarimeter apparatus through which $365 \mathrm{~nm}$ wavelength light can pass, having its polarization measured before and after the passage through the protein matter. Liquid connected to a temperature bath can flow through the sample holder around and outside the inner tube. We used a light-viscosity silicone oil (Dow Corning 200 fluid, $1.2 \mathrm{cP}$ ) as the heat bath liquid since it is transparent to microwaves. Both the polarization and the temperature can be monitored simultaneously.

The experimental procedure is to start measuring the polarization of the cold denatured state at about $5^{\circ} \mathrm{C}$ after a long equilibration time $(3 \mathrm{~h})$. The temperature is subsequently stepped in increments of $2^{\circ}$ until about $44^{\circ} \mathrm{C}$. At each measurement point, time was allowed for temperature equilibration (in total $5 \mathrm{~min}$ ), but not for the much slower equilibration of the $\beta$-lactoglobin sample. For this reason upon heating and subsequent cooling an effective hysteresis loop exists caused by the slow kinetics.

The polarization variable $\alpha$, which we monitor on a polarimeter (Perkin-Elmer), is related to the specific rotation $(\alpha)_{\lambda}$ at a given wavelength $\lambda$ by [11]

$$
(\alpha)_{\lambda}=\frac{100}{c d} \alpha
$$

where $d$ is the optical path length through the sample in decimeters and $c$ is the concentration of protein in $\mathrm{g} / 100 \mathrm{ml}$. The specific rotation can in turn be related to the structural properties of the protein molecule and, to some extent also, to the solution, by the equation [12]

$$
(\alpha)_{\lambda}=\frac{3 M}{100\left(n^{2}+2\right)}\left(\frac{a_{0} \lambda_{0}^{2}}{\lambda^{2}-\lambda_{0}^{2}}+\frac{b_{0} \lambda_{0}^{2}}{\left(\lambda^{2}-\lambda_{0}^{2}\right)^{2}}\right) \text {, }
$$




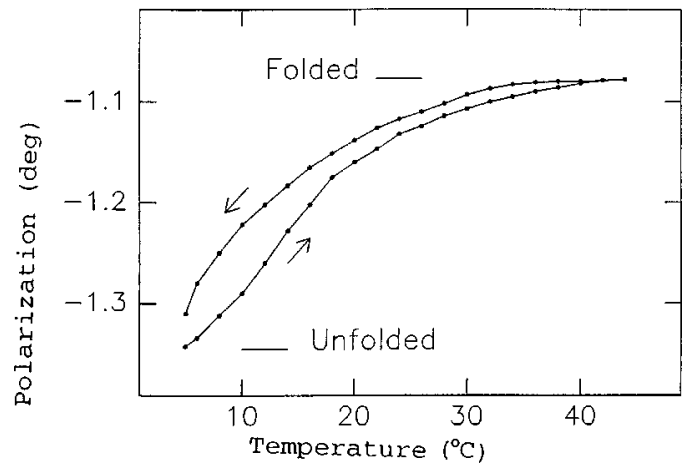

FIG. 1. Polarization data for a kinetic loop of thermal folding and unfolding of $\beta$-lactoglobulin. The arrows indicate the direction of the temperature cycle. The polarization values for the folded and unfolded states are indicated on the figure.

where $M$ is the mean residue weight and $n$ is the refractive index of the medium at wavelength $\lambda$. In the last equation the constants $b_{0}$ and $\lambda_{0}$ are sensitive to structural properties such as the helical conformation of the protein molecule but insensitive to solvent effects. The constant $a_{0}$ is sensitive to the conformation of the polypeptide chain and to the medium. $\lambda_{0}$ is a dispersion constant.

\section{RESULTS}

$\beta$-lactoglobulin was chosen as the test protein because of the accessibility of the cold denaturated, folded, and hot denaturated states. In a $4 M$ urea solution the protein undergoes a transition from cold denaturation to the folded state around $20{ }^{\circ} \mathrm{C}$. The protein is derived from cow milk and has a barrel-like structure. The pronounced content of secondary structure elements is sensitive to polarization measurements as obtained from the ORD experiment. Hence, working with $\beta$-lactoglobulin provides the advantage of working with the easily accessible cold denaturation phase so that all refolding experiments can exclusively deal with cold denaturation and avoid hot denaturation, which often involves protein aggregation.

The basic refolding experiment for $\beta$-lactoglobulin consists of bringing the sample from the cold-denaturated state over to the folded state by raising the temperature from 5 to $44^{\circ} \mathrm{C}$ and then back again to the cold denaturated state at $5{ }^{\circ} \mathrm{C}$, where the system is allowed to equilibrate completely over about three hours. When this refolding experiment is done during a matter of a few hours the heating-cooling cycle can be monitored as a kinetic loop (Fig. 1). If the measurements at each temperature step are performed with the sample at perfect equilibrium (equilibrium between the concentrations of protein in the denatured and folded states) the loop will collapse to a line of polarization values as a function of temperature. The appearance of a kinetic hysteresis loop is a result of the slow reaction kinetics, and measurement of the loop therefore allows the study of abrupt changes in the reaction kinetics. The temperature can be monitored inside the sample holder of the polarimeter by a thermocouple wire.

As is commonly done for proteins, one can assume that the transition from the native folded state to the denatured state involves formation of unstable intermediates in a two- state process, and characterized by a variable $f_{D}$ measuring the fraction of molecules in the denatured state $D$. The experimental value $\alpha$ (the polarization) that monitors the transition from the native state $N$ to the denatured state $D$ can be expressed in terms of the initial and final state values, respectively $\alpha_{N}$ and $\alpha_{D}$, as

$$
\alpha=\left(1-f_{D}\right) \alpha_{N}+f_{D} \alpha_{D},
$$

and the equilibrium constant for the reaction $N \rightarrow D$ can be written as

$$
K=f_{D} /\left(1-f_{D}\right)=\frac{|\alpha|-\left|\alpha_{N}\right|}{\left|\alpha_{D}\right|-|\alpha|},
$$

which can be determined at each temperature during the heating/cooling cycle of the refolding/denaturation process. The heating/cooling cycle is monitored by the polarization $\alpha_{P}=\alpha / 200+100$ changing from about 98.70 to about 98.90 , corresponding to a change in the equilibrium constant $K$ from 1.43 to about 0.30 , and which again corresponds to an enthalpy change $\Delta H_{D}$ from $-181.5 \mathrm{~kJ} / \mathrm{mol}$ to about 11.0 $\mathrm{kJ} / \mathrm{mol}$

\section{Refolding of $\beta$-lactoglobulin with microwaves}

In the next series of experiments the previously described heating/cooling cycles are studied when microwave irradiation is applied to a protein sample at certain temperatures during the refolding and denaturation process.

The microwave radiation was provided from a standard magnetron of a microwave oven operating at $2.45 \mathrm{GHz}$ and with a power of $800 \mathrm{~W}$. The microwave oven was remodeled so that it could operate on the sample holder of the polarimeter while it was constantly in contact with the heat bath with a circulating silicone liquid. Inside the oven several containers were placed, containing in all 21 of water, in order to absorb some of the microwave power released during the operation. When microwaves are applied, the sample holder is inserted into the microwave oven without disconnecting it from the heat bath. The temperature is monitored before and after microwave irradiation. The microwave irradiation is applied for $5 \mathrm{~s}$ at $800 \mathrm{~W}$, causing a temperature increase of the protein sample of about $0.3^{\circ} \mathrm{C}$. The power absorbed by the 3 $\mathrm{ml}$ sample was about $0.75 \mathrm{~W}$. After the microwave irradiation the sample holder is quickly put back into the polarimeter and the polarization is remeasured.

The experiment from before is repeated starting with the denatured $\beta$-lactoglobulin solution equilibrated at $4{ }^{\circ} \mathrm{C}$. First the temperature is increased to $48{ }^{\circ} \mathrm{C}$ without the application of microwaves. Then the temperature is lowered back to $4{ }^{\circ} \mathrm{C}$; during the decrease at $20^{\circ} \mathrm{C}$ microwaves are applied. After reequilibration at $4{ }^{\circ} \mathrm{C}$ the temperature is raised to $48^{\circ} \mathrm{C}$ again. At $8^{\circ} \mathrm{C}$ during the increase microwaves are applied. The results are shown in Fig. 2. The small difference in the polarization values when compared to Fig. 1 is due to a slight difference in protein concentration in the sample.

It can be seen that at the microwave irradiation at $8{ }^{\circ} \mathrm{C}$ the polarization makes a large jump toward the folded state. Temperature effects can better be separated from microwave effects during the cooling cycle. During the cooling cycle microwave power was applied at $20^{\circ} \mathrm{C}$ and here we obtained 


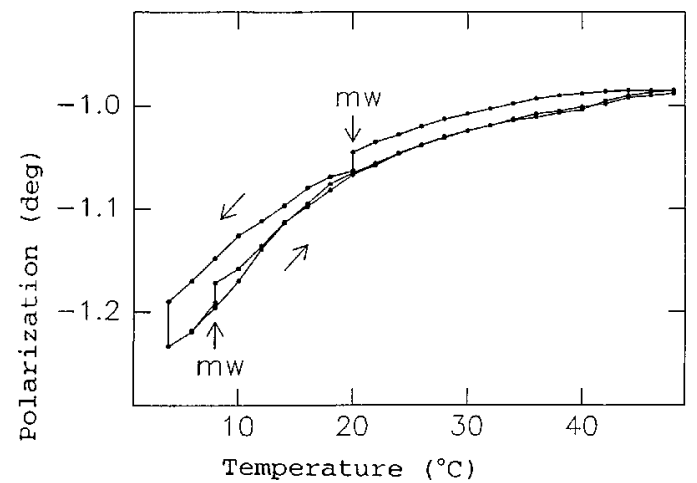

FIG. 2. Polarization data for a kinetic loop of folding and unfolding of $\beta$-lactoglobulin where microwaves are applied. Arrows indicates the direction of the cycle, and arrows marked "mw" indicate the temperature at which microwaves where applied.

a large jump downward toward the denatured state (Fig. 2). Applying microwaves apparently enhances the reaction kinetics and hence follows the trends of the heating/cooling schedule. Therefore the microwave treatment of the proteins in solution seems to be nonthermal. This microwave effect was reproducible.

These effects of microwave irradiation on the protein solution might at first seem small. However, when one considers the rate of change by which the protein in solution is approaching its equilibrium state between the folded and unfolded structures, a large difference in the corresponding rate constant is observed. Without microwave irradiation it takes on the order of hours to reach equilibrium while with microwaves the equilibrium at that temperature is reached on the order of seconds and faster than $1 \mathrm{~min}$. This is nicely illustrated in Fig. 3(b) where the polarization of a refolding experiment with $\beta$-lactoglobulin is measured in time at a fixed temperature with and without microwave irradiation. Hence, according to these time measurements, the effects are two orders of magnitude (a factor of 100) or more on the folding kinetics.

Additional experiments have been carried out along with this heating/cooling cycle. One "blind" experiment is a repetition of the procedure without switching on the microwave oven, another is without protein in the solution. In neither case were any effects observed on the polarization data. We also performed local small heating/cooling cycles, involving temperature changes of up to $3^{\circ}$, within the large heating/cooling cycle. This was done in order to check for any effect similar to that of the microwave irradiation. Although these thermal cycles could not be done as quickly as the microwave irradiation, the experiments could not reproduce the microwave data, as shown in Fig. 3(a). This therefore helps to demonstrate the nonthermal nature of the observed effects.

Other globular proteins have also been used in the same type of experiment as described above. One of these was ribonuclease (from horse), which is a very well-known protein with respect to refolding experiments. Since the protein folds faster than $\beta$-lactoglobulin, one does not see as pronounced a hysteresis effect in the heating/cooling curve. However, the effect of microwave irradiation was as pronounced as that for $\beta$-lactoglobulin. Also, other regions of frequencies for the optical rotation were tried, giving still the same effect of microwave irradiation. We have, for example, repeated the refolding experiment at $304 \mathrm{~nm}$ and get the same type of results as shown in Figs. 1 and 2 but with more sensitivity to secondary structure formation.

\section{COHERENT MODES IN THE GHz RANGE ON CHAIN MOLECULES}

One possible cause of the effect of microwave irradiation on protein folding might be the excitation of collective intrinsic modes in the protein. We have estimated the frequency of intrinsic torsional modes and shown that they are well matched to technical/commercial microwave sources $[5,6]$.

In physical and biological systems interactions of biological chain molecules take place with the aqueous environment, with other chain molecules, with membranes, etc. For closed chain molecules, such as circular DNA, the topological conservation of White [13] implies that the sum of the torsion and the writhing is constraint to a constant power (the linking number). Due to the finite viscosity of the aqueous medium, proteins are topologically restricted when considered on a sufficiently short time scale, a fraction of a microsecond and less. The torsional term can be considered separately as there is not sufficient time for occurrence of the writhing term in the White equation [13-15]. Coherent intrinsic motion of higher frequencies can therefore take place.

The eigenfrequency $\nu$ can be estimated from a simple calculation of the energy, with a rotational and torsional contribution as follows:

$$
E=E_{\mathrm{rot}}+E_{\mathrm{tor}}=\frac{1}{2} I\left(\frac{\partial \phi(\sigma)}{\partial t}\right)^{2}+\frac{1}{2} \alpha\left(\frac{\partial \phi(\sigma)}{\partial \sigma}\right)^{2},
$$

where $\sigma$ is a spatial variable along the backbone chain and $\phi(\sigma)$ is a vector field orthogonal to the chain axis measuring the displacement. $I$ is the moment of inertia and $\alpha$ is the torsional constant. The Euler-Lagrange equation is

$$
\left(I \frac{\partial^{2}}{\partial t^{2}}-\alpha \frac{\partial^{2}}{\partial \sigma^{2}}\right) \phi(t, \sigma)=0 .
$$

This equation is solved with the well-known wave ansatz $\phi(\sigma-v t)$, where the velocity $v$ is given by

$$
v=\sqrt{\alpha / I} \text {. }
$$

The eigenfrequency $\nu$ is then derived from the velocity $v$ as

$$
\nu=\frac{1}{L} \sqrt{y / i},
$$

where $L$ is the length of the chain molecule that participates in one period of the torsional mode, $y(\alpha)$ is the torsion constant per inverse unit length, and $i$ is the moment of inertia of the back-bone per unit length. Typical values are not easy to obtain. For proteins we may take a rotation of $\pi / 2$ to correspond to about $1 \mathrm{eV} / \AA$, in agreement with common bond energies, although the energy involved in strained chemical bonds is often smaller. Hence, the torsion constant per inverse unit length, $y$, is about $0.4 \mathrm{eV} / \AA$. The moment of inertia of the backbone (per unit length), $i$, is about 100 a.u. $\AA$, 

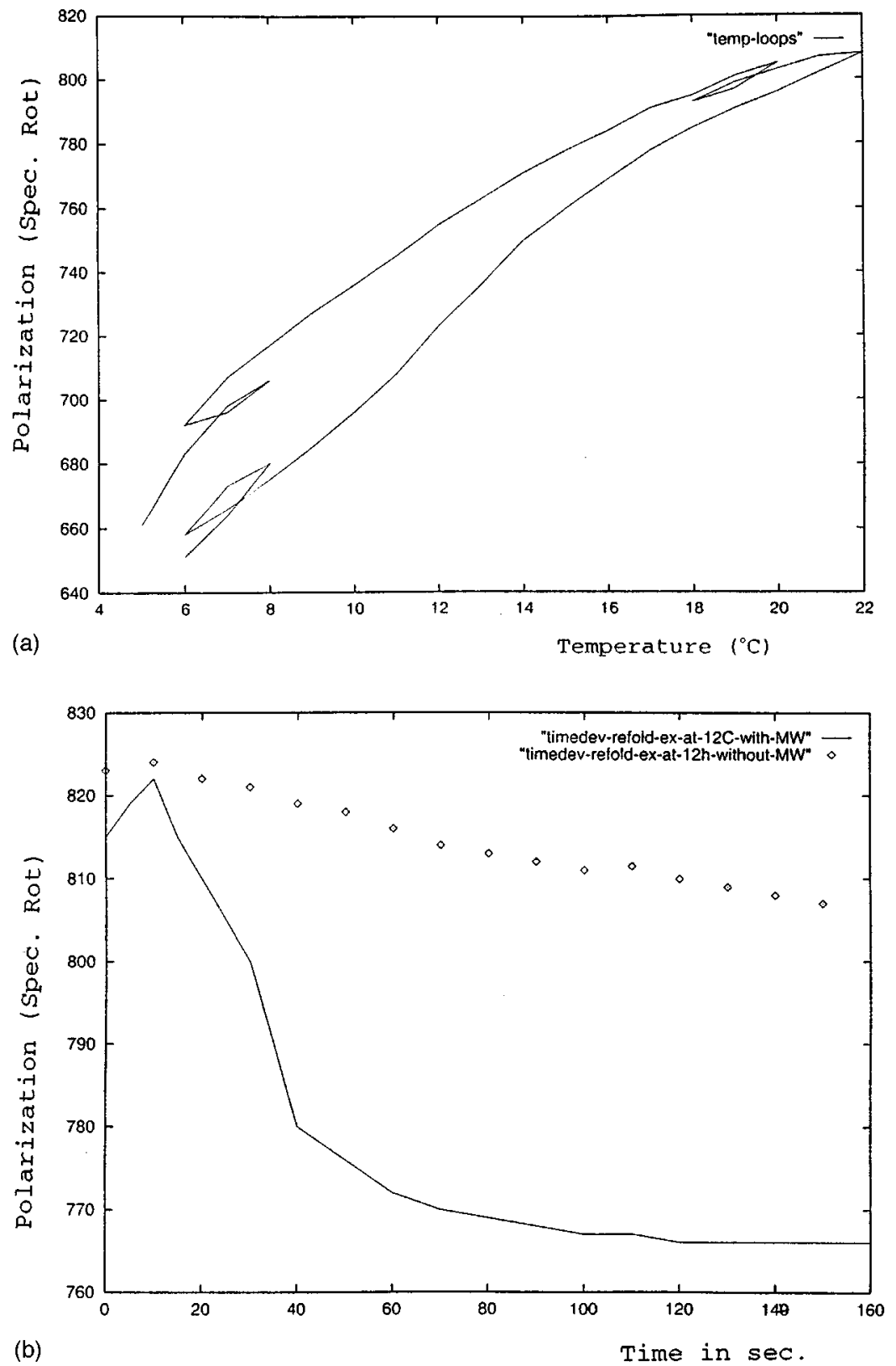

FIG. 3. (a) Temperature loops performed on the same experiment as in Fig. 1. The temperature loops are done in, e.g., the refolding process, by raising the temperature from $t_{0}$ by $1{ }^{\circ} \mathrm{C}$, measuring, and then lowering the temperature by $2{ }^{\circ} \mathrm{C}$, measuring, and raising it back to $t_{0}$. These loops are clearly different from the microwave jumps. (b) The time development of the polarization at $12{ }^{\circ} \mathrm{C}$ before and after applying microwaves (MW). Before, the equilibration is a matter of hours, after, it is clearly a matter of seconds. On the ordinate is shown the polarization and on the abscissa the time from 0 to $150 \mathrm{~s}$ after the temperature is fixed. The diamonds are before and the full line after application of MW. After 1 min equilibrium is achieved and the curve is becoming horizontal. depending on the degree to which the sidechains are involved in the torsion. A typical value for $L$ is $475 \AA$, and hence the frequency $\nu$ becomes about $8 \mathrm{GHz}$. This estimate is based on the assumption of a linear torsion term; however, as the torsion term is not linear the estimate is only an upper limit. We think that possibly it is a factor of 10 too large. It should further be noticed that the width of the resonance will be very large because of the dispersion. As can be seen, the frequency of such coherent modes in biomolecular chain molecules turns out to be of the same order of magnitude as those typical of radio- and microwave applications.

\section{REACTION KINETICS OF PROTEIN FOLDING AND TUNNELING}

Let us for simplicity consider the folding transition as a one-step process over an energy barrier A. As in Sec. III we consider the transition from the denatured phase $D$ to the folded, native phase $N$ of a protein: $N \rightarrow D$.
It is further assumed that the transition is caused by the instability (to tunneling) of one dominating mode. The transition rate can then be approximated as the transition integral for tunneling multiplied by the frequency of the responsible mode. This mode was first considered by Beiser [16] in connection with an estimate of $\alpha$ decay rates. The rate constant for protein folding is thus proportional to

$$
k \simeq \omega_{\text {mode }} e^{-A / k_{B} T} .
$$

The factor $e^{-A / k_{B} T}$ stems from the transition integral

$$
\mathcal{T} \simeq \exp \left(-\frac{2}{\hbar} \sqrt{2 m_{\text {eff }}} \int \sqrt{U_{\text {conf }}(x)-U_{D} d x}\right)
$$

where $m_{\text {eff }}$ is the effective mass in connection with the movements and bending of the protein chain. $U_{\text {conf }}$ and $U_{D}$ are respectively the internal energy of a conformation of the folded protein (one can take a helix conformation on a lat- 
tice) and the internal energy of the denatured state of the protein. The result in Eq. (9) then arises from an expansion of the square root.

\section{MICROWAVE IRRADIATION EFFECTS ON REACTION KINETICS}

As we discussed previously, applying microwaves apparently enhances the reaction kinetics and hence follows the trends of the heating/cooling schedule. Furthermore, the microwave treatment of the proteins in solution seems to be nonthermal, a result that was reproducible and tested through blind experiments.

The effects of microwave irradiation on the protein solution might at first seem small. However, when one considers the rate of change by which the protein in solution is approaching its equilibrium state between the folded and unfolded structures, a large difference in the corresponding rate constant is observed. Without microwave irradiation it takes on the order of hours to reach equilibrium while with microwaves the equilibrium at that temperature is reached in less than $10 \mathrm{~s}$. These effects are thus two orders of magnitude (a factor of 100) or more on the folding kinetics.

How can we explain this effect of microwave irradiation on the folding kinetics? We imagine again that the folding kinetics going from a denatured state $D$ to the folded, native state $N$ involves crossing a barrier of height $A$. Let $B$ be the amplitude of the applied electromagnetic radiation with frequency $\omega$. With application of microwave irradiation intrinsic modes can be excited in a resonance fashion. Such cross sections for excitation have long tails around the resonance peak due to dispersion. Microwave absorbtion by water is, for example, significantly displaced by several decades away from the rotational frequency. The effects of resonant ab- sorption are to lower the barrier for tunneling and to increase the coherence of the intrinsic motions. Because of the first effect, the transition amplitude and thus the rate constant are expected to depend on the amplitude of the applied electromagnetic field.

Therefore, by applying external electromagnetic radiation close to the frequency of an intrinsic mode, we can expect an enhancement of the kinetics. In our case the intrinsic modes were torsional modes. Standard microwave ovens with a frequency of $2.45 \mathrm{GHz}$ were chosen because this frequency is close to that calculated above for torsional modes.

\section{CONCLUSION}

To conclude, refolding experiments on $\beta$-lactoglobulin with applied microwave radiation show that there are nonthermal effects of microwave irradiation that can enhance the kinetics of the folding and denaturation processes. The observed effects of microwaves on $\beta$-lactoglobulin were consistently reproducible in repeated experiments on both that protein and other globular proteins. The effects observed in this paper of being able to change the processes of folding and denaturation by external radiation and perhaps even steer the reactions could be promising for future applications in biotechnology, such as recombinant DNA techniques, and may help to further the development of understanding of the interaction of electromagnetic radiation with biological systems.

\section{ACKNOWLEDGMENTS}

Scientists and technical staff at the Institute of Chemistry and the Institute of Physics at DTU are acknowledged for their help in carrying out the experiments.
[1] T. E. Creighton, Proteins, 2nd ed. (Freeman, New York, 1993).

[2] J. D. Bryngelson and P. G. Wolynes, Proc. Natl. Acad. Sci. USA 84, 7524 (1987).

[3] A. Sali, E. Shahknovich, and M. Karplus, Nature (London) 369, 248 (1994).

[4] K. A. Dill, S. Bromberg, K. Yue, K. M. Fiebig, D. P. Yee, P. D. Thomas, and H. S. Chan, Protein Sci. 4, 561 (1995).

[5] J. Bohr, H. Bohr, and S. Brunak, Biophys. Chem. 63, 97 (1997).

[6] J. Bohr and H. Bohr, Z. Phys. D: At., Mol. Clusters 40, 186 (1997).

[7] J. L. Sagripanti, M. L. Swicord, and C. C. Davis, Radiat. Res. 110, 219 (1987).
[8] C. Y. Wu, S. T. Chen, S. H. Chiou, and K. T. Wang, J. Protein Chem. 11, 45 (1992).

[9] A. Morales-Rubio, C. J. Salvador, and M. de la Guardia, Microchemical J. 47, 270 (1993).

[10] G. M. Reynolds, F. I. Young, J. A. Young, A. Williams, and D. C. Rowlands, Cytopathology 5, 345 (1994).

[11] G. Weber and F. W. J. Teale, in The Proteins, Composition, Structure and Function, 2nd ed., edited by Hans Neurath (Academic, New York, 1965), Chap. 17, pp. 445-521.

[12] W. J. Moffit, Chem. Phys. 25, 467 (1956).

[13] J. H. White, Am. J. Math. 91, 693 (1969).

[14] F. B. Fuller, Proc. Natl. Acad. Sci. USA 68, 815 (1971).

[15] F. H. Crick, Proc. Natl. Acad. Sci. USA 73, 2639 (1976).

[16] Arthur Beiser, Concepts of Modern Physics, 5th ed. (McGrawHill, New York, 1995). 